\title{
Du cannabis sous les cacaoyers : épuisement du modèle pionnier et reproduction des « institutions de la frontière " en Côte d'Ivoire forestière
}

Oléagineux, Corps Gras, Lipides. Volume 8, Numéro 6, 611-20, Novembre - Décembre 2001, Dossier : L'avenir des cultures pérennes

Auteur(s) : Eric LEONARD, Ciesas-Golfo, avenida Encanto esq. A. Nava, colonia El Mirador, CP 91 170, Xalapa, Veracruz, Mexique.

Résumé : Structuration du milieu rural de la zone forestière ivoirienne : rôle de la "frontière interne » et de ses institutions : Kopytoff [1] puis, en référence au contexte ivoirien, Chauveau [2] ont montré à quel point les phénomènes de "frontière interne " ont joué un rôle structurant dans la constitution des espaces nationaux et des champs identitaire, politique et économique en Afrique subsaharienne. En Côte d'Ivoire, l'économie de plantation de café et de cacao, à travers les fronts pionniers qui ont balayé d'est en ouest la moitié méridionale du pays, a ainsi largement contribué au façonnage du système politique et de l'ensemble de la société civile [3]. Au point que la crise économique, politique et institutionnelle que traverse la Côte d'Ivoire depuis la fin des années 80 se superpose dans une large mesure à la crise du secteur exportateur de café et de cacao, elle-même indissociable de l'épuisement des frontières internes et des difficultés que pose la reproduction des systèmes d'exploitation pionniers (voir, entre autres, les lectures de cette crise que proposent Contamin et Memel-Fotê [4] ; Losch [5] ; Ruf [6]). Parmi ces « institutions de la frontière » qui ont tant pesé dans la construction d'un "État paysan » en Côte d'Ivoire et dans l'élaboration d'un mode de gouvernance aujourd'hui en crise [2], celles qui ont encadré la mise en rapport des deux principaux facteurs du boom de l'arboriculture d'exportation - la terre forestière et le travail fourni par les migrants - retiennent d'emblée l'attention, en ce sens qu'elles ont été le véritable support des dynamiques pionnières et ont structuré de façon durable les relations entre les différents acteurs de la dynamique de colonisation - paysans de différentes origines, autorités coutumières, commerçants, représentants des administrations publiques. En particulier, les différentes formes de " tutorat " entre les paysans déjà établis, autochtones ou allogènes, et les nouveaux arrivants ainsi que les échanges de prestations auxquels elles renvoient ont profondément marqué les processus d'installation sur la frontière agricole et d'expansion de la frontière agricole. Elles ont également modelé l'organisation et le fonctionnement des exploitations agricoles en permettant de différer et, souvent, de minorer la rémunération du travail de mise en place et d'entretien des plantations pérennes, en même temps qu'elles institutionnalisaient la prestation de services récurrents au bénéfice des aînés sociaux, bailleurs de la terre. Avec l'épuisement des frontières internes et l'impossibilité de procéder aux échanges de terre et de travail qui fondaient les rapports de tutorat, c'est toutes les relations d'allégeance et de clientélisme entre les différentes composantes de la société pionnière (autochtones et allochtones, aînés et cadets de la migration, paysans et représentants de l'État) qui se trouvent remises en question et, avec elles, les fondements même de l'organisation et de la reproduction des exploitations agricoles, sans que la dépression durable des cours internationaux du café et du cacao permette l'expression et la diffusion d'innovations institutionnelles pouvant se superposer et, a fortiori, se substituer aux précédentes. Cette crise des institutions agraires de la frontière semble avoir une incidence directe sur la remise en question des fondements institutionnels du système ivoirien de régulation sociopolitique, à en juger par la position centrale des dynamiques rurales, en général, et des tensions foncières, en 
particulier, dans le débat politique contemporain et les polémiques sur l'« ivoirité » ou la place allouée aux " étrangers » dans les champs économique et politique nationaux. Le développement récent de la culture et du trafic de cannabis dans les anciens fronts pionniers cacaoyers du Sud-Ouest, dans la mesure où il coïncide avec l'épuisement du modèle pionnier de mise en valeur du milieu et de régulation socio-économique, appelle une lecture attentive des dynamiques institutionnelles qui ont supporté son essor. Il apparaît, en effet, que l'organisation de la production de marihuana et, plus largement, celle de l'ensemble de la filière illicite sont venues soutenir, au moins durant la première moitié des années 90 , le fonctionnement des institutions relevant du tutorat et les relations de type clientéliste qui en dérivent : l'innovation technique - l'intégration d'une nouvelle culture dans les systèmes de production - semble en l'occurrence avoir suppléé la dynamique d'innovations institutionnelles dans le champ de la régulation foncière, en permettant la reconduction de rapports sociaux spécifiques de la phase pionnière que l'épuisement de la frontière agricole semblait condamner. De façon plus générale, la production et le commerce de la marihuana semblent avoir fourni un support économique essentiel à des organisations qui avaient accompagné et soutenu le développement des fronts pionniers, non seulement dans le domaine de la production agricole mais aussi dans celui de la mise sur le marché aux niveaux national et international. Les filières de commercialisation intérieure du café et du cacao, durement touchées au cours des années 90 par la restructuration du système de régulation étatique et par l'assèchement des crédits bancaires, semblent, en particulier, avoir rencontré dans le développement des activités illicites un soutien financier que la sphère légale ne pouvait plus leur fournir 1. L'économie du cannabis a ainsi constitué durant la dernière décennie un facteur d'amortissement des crises de différente nature (financière, écologique, sociale) qui remettaient en question la reproduction même du tissu d'exploitations agricoles et de relations sociales de tous types constituant la base de l'économie de plantation ivoirienne.

Summary : After being the scene of a huge cocoa boom based on the development of a large pioneer front that swept the forest part of the country from East to West, Côte d'Ivoire is now facing a deep economical, ecological, and political crisis. In many respects, this crisis responds to a crisis of the pioneer farming systems and; more specifically, to the crisis of the agrarian institutions that regulated the access to both land and labour factors as well as to the coffee and cocoa markets and constituted powerful tools for social regulation. The spread of cannabis cultivation and marihuana trafficking since the end of the 1980s must be considered in connection with this crisis of the pioneer farming systems and institutions. This paper shows how the spread of cannabis cultivation has been resting on those pioneer-specific institutions and is enabling their reproduction, as if the technological innovation (the introduction of a new product into the farming and marketing systems) were allowing the social adjustments that the ecological and economical crisis seemed to dictate to be postponed.

Keywords : social regulation, land access, frontier institutions, clientelism, drug trafficking.

\section{ARTICLE}

Dynamique pionnière et contrôle de la force de travail : la construction d'institutions spécifiques, supports de rapports clientélistes

La dynamique de colonisation agraire et le développement d'une économie de plantation paysanne ont donc conduit à la mise en place d'institutions spécifiques, résultant des cadres social et légal (le 
statut juridique des terres colonisées, la mise en application des règles officielles par les représentants locaux de l'administration et les dispositifs locaux de gestion de ces terres) qui ont présidé au processus de frontière, ainsi que des conditions agroécologiques et des options techniques qui ont pesé sur l'organisation des exploitations agricoles. II n'est pas de mise ici de détailler l'ensemble des facteurs qui ont modelé les dynamiques institutionnelles sur la frontière, mais il faut évoquer brièvement les contraintes en termes d'accès au foncier et au travail qui ont orienté le comportement des acteurs. En matière de réglementation foncière, la substitution d'un projet de loi (jamais promulgué) par le slogan " la terre appartient à celui qui la met en valeur ", lancé par le président Houphouët-Boigny en 1963, a fixé le cadre normatif de la colonisation agricole ${ }^{2}$ : la superposition des droits coutumiers et d'une norme officielle, dénuée de fondement légal mais ayant force de loi, a conduit à une gestion de proximité et pragmatique du foncier rural, sur des bases clientélistes et le plus souvent au bénéfice des colons agricoles [2].

Sur la frontière agricole du Sud-Ouest, dans un contexte marqué par de faibles densités de population autochtone, les fortes disponibilités foncières et l'incapacité des détenteurs coutumiers d'en réguler I'accès autrement que par l'aliénation et l'établissement de relations de tutorat, la force de travail a constitué le premier facteur limitant de l'accumulation, tant pour les autochtones que pour les nouveaux arrivants. Comme le précise Ruf [7], devant le niveau technique relativement homogène des exploitations et « faute de pouvoir augmenter la productivité du travail et donc les plus-values tirées de chaque actif, l'accumulation ne peut passer que par une multiplication du nombre d'actifs » (7 : T50) ; au-delà de l'objectif d'appropriation de la terre, l'enjeu principal a donc été de capter le plus de main-d'œuvre possible. Les stratégies de l'ensemble des acteurs en concurrence pour la maîtrise de l'espace et sa mise en valeur ont donc visé la construction de « marchés captifs » de travail.

"Étrangers ", "six-mois " et " petits-frères " : le rapport de tutorat, institution centrale dans la dynamique pionnière

Deux caractéristiques antagoniques orientent dans ce domaine les négociations autour de la captation de main-d'œuvre et des arrangements institutionnels qui en résultent. D'une part, l'offre de travail sur les fronts pionniers est loin de répondre aux mécanismes d'un marché ouvert : I'organisation de la migration et les logiques de minimisation des coûts de transaction de la part de migrants concourent au cloisonnement de cette offre sur une base ethnique, clanique ou familiale. D'autre part, les fortes disponibilités foncières, au niveau de la zone de colonisation ou plus avant sur la frontière agricole, limitent la capacité des détenteurs de terre, traditionnels autant que pionniers de la première génération, à construire de véritables monopoles d'emploi, au moins tant que les limites physiques de l'espace à coloniser ne sont pas atteintes.

Ces contraintes ont conduit à l'élaboration de divers arrangements institutionnels reposant communément sur l'échange de terre contre du travail et permettant aux nouveaux arrivants d'accéder au foncier à l'issue d'une période plus ou moins longue de travail chez un planteur. Pour les pionniers comme pour les autochtones, en effet, la meilleure façon de valoriser les terres qu'ils ne peuvent rapidement exploiter, dans un contexte de course à la ressource et de faible reconnaissance des droits fonciers sur les espaces non cultivés, est encore d'en utiliser une partie pour s'attacher une main-d'œuvre clientélisée, avant que ne se l'approprient d'autres migrants ou ne la vendent d'autres ressortissants autochtones. Au-delà du contenu explicite des contrats de travail (à la tâche, mensualisation, par partage de la récolte, incluant ou non l'alimentation, les charges de santé et I'hébergement du travailleur) et du salaire "immédiat " qui leur correspond, ces arrangements 
institutionnels sous-tendent donc fréquemment un accord implicite des deux parties autour du versement d'un salaire différé, sous la forme d'un lopin de terre ; ils impliquent par ailleurs, sous couvert du principe de réciprocité, une dette morale liant le travailleur à celui qui lui a procuré une terre, dette formalisée par la fourniture de redevances plus ou moins régulières, généralement en travail, et contenue dans le rapport de « tutorat » ou de patronage.

Voici donc établi le " socle commun ", transverse aux différents groupes socioculturels, des contrats de travail qui se formalisent lors de la phase pionnière. D'un groupe à l'autre, leur contenu pratique varie et, avec lui, le montant du salaire immédiat, celui du "salaire " différé ainsi que le délai de versement de ce dernier. Ces variations sont à l'origine des mécanismes d'accumulation différentielle observables entre les différents groupes ainsi qu'au sein de ces groupes [6-8]. Certains arrangements sont communs à l'ensemble de la société agraire, d'autres sont spécifiques à tel ou tel groupe. Dans la première catégorie, on peut classer les contrats salariés établis sur une base annuelle ou mensuelle, ainsi que ceux qui donnent lieu au partage de la récolte, généralement au tiers en faveur du manœuvre (abu-san) ${ }^{3}$; ces contrats sont particulièrement répandus chez les planteurs qui ont mis en valeur la quasi-totalité de la surface qu'ils avaient acquise et servent souvent de base d'insertion pour des travailleurs dont la migration a été peu « encadrée " par des structures claniques ou familiales. Les conditions ultérieures d'accès du migrant à la terre demeurent de ce fait sujettes à controverse, puisqu'elles reposent davantage sur les capacités de médiation de l'employeur que sur ses propres disponibilités foncières, et confèrent à ces contrats une instabilité que souligne la forte mobilité des travailleurs d'une exploitation à l'autre.

La seconde catégorie regroupe des arrangements qui sont plus spécifiques à tel ou tel groupe et qui constituent de ce fait des indicateurs de la capacité de chacun de pratiquer des taux élevés d'exploitation de sa main-d'œuvre salariée. À cet égard, les autochtones semblent être dans une situation particulièrement défavorable. Plus encore que dans le Centre-Ouest, où les villageois étaient parvenus à fixer temporairement des métayers dyula et voltaïques préalablement à leur accès aux terres, les autochtones bakwé, kodia et kru du Sud-Ouest ont généralement été conduits à aliéner les leurs sans autres contreparties - au-delà du versement d'une somme d'argent généralement bien inférieure à celle qui avait été convenue - que l'affirmation de la relation de " tutorat " et les contreprestations, souvent symboliques, qui lui sont associées. Le tutorat peut cependant être considéré comme l'une des institutions centrales du fonctionnement de la société pionnière, en cela qu'il fait l'objet d'une reconnaissance générale de la part des colons et que les "obligations " qu'il implique (contribution aux problèmes financiers du tuteur, tant pour des motifs " structurels » - scolarisation que pour des causes conjoncturelles - funérailles, divorce) sont généralement admises par ceux-ci et, parfois, par leurs descendants, dès lors que les exigences des autochtones demeurent limitées ([2] p. 106-7). Les clientèles qui en résultent sont cependant friables et les transferts de services demeurent trop aléatoires pour servir de base à une stratégie de capitalisation par les plantations : l'accès à la main-d'œuvre a lieu, dès lors, à prix coûtant et doit être financé par de nouvelles cessions de terre.

Les formes de tutorat ou de patronage qui s'élaborent au sein des sociétés de migrants sont en revanche plus structurantes et donnent lieu à des rapports d'exploitation beaucoup plus systématiques et contraignants pour les cadets sociaux. Il en va ainsi des modalités d'accès à la terre, qui reposent dans les communautés baoulé et burkinabé sur les institutions du " six-mois " et du " petit-frère ", respectivement. Dans les deux cas, le processus migratoire aboutit à l'insertion dans un tissu social fortement hiérarchisé, au sein duquel l'antériorité de l'installation détermine la prééminence et les prérogatives en matière de prélèvement du surtravail des nouveaux arrivants. Chez 
les Baoulé notamment, les chefs historiques de la migration se sont arrogés les facultés de maître de la terre pour les déléguer à des chefs de deuxième ou troisième niveau (chef de carrefour, chef de piste, chef de campement), chargés d'organiser les cellules de défrichement $[9,10]$. L'un des privilèges de ces chefs réside dans leur légitimité à convoquer des prestations exceptionnelles (ukalè), à travers lesquelles tous les cadets sociaux sont astreints de leur fournir un travail gratuit et sans contrepartie. Selon Lesourd ([10] p. 85), ces prestations ont pu représenter jusqu'à $30 \%$ des défrichements des plus grands planteurs sur le front pionnier de Méadji.

Les performances remarquables des Baoulé en ce domaine sont cependant étroitement liées au système d'organisation des arrivées par le biais de l'institution du " six-mois ". II s'agit d'un contrat dont la durée et les modalités permettent une utilisation optimale de la main-d'œuvre juvénile, à un taux d'exploitation relativement élevé ${ }^{4}$, entre deux exploitations : I'une située en zone forestière, qui héberge les travailleurs au cours des pointes de travail sur les cacaoyères (sarclages et traite), entre juin et décembre, et une autre, en zone de savane, où les calendriers de culture de l'igname permettent de récupérer cette main-d'œuvre au moment des travaux de récolte et de buttage. Les modalités pratiques de ces contrats (taux de rémunération, nombre d'hommes et de femmes ${ }^{5}$, dates et durée de l'emploi) sont négociées entre les deux chefs d'exploitation, qui sont souvent des parents (oncle/neveu, cousins), hors de toute médiation des travailleurs. Surtout, le contrat de " six-mois " apparaît comme l'institution qui prépare et supporte la migration de colonisation des Baoulé ; elle tient lieu de rite probatoire, préalable à la cession de terre. En ce sens, et bien qu'il provienne de l'organisation des exploitations baoulé dans la région d'origine, le rapport de " six-mois » peut être considéré comme une " institution de la frontière agricole ».

L'encadrement de la migration entre des cellules familiales apparentées permet de reconstruire sur la frontière agricole la structure de la société d'origine, qui intègre enfants, neveux, frères et cousins [12]. Les neveux, en particulier, constituent un volant de main-d'œuvre gratuite - au coût de son alimentation près - ou rémunérée au tiers, dans l'attente de leur installation. Une enquête réalisée en 1994 sur le front pionnier de Méadji fait ainsi apparaître que 88 \% des Baoulé résidant dans la région sont arrivés chez un parent direct (père, oncle, cousin), cette proportion tendant à s'accroître dans les générations récentes : $95 \%$ chez les moins de 35 ans contre $80 \%$ pour les planteurs de plus de 50 ans ([13] p. 163). Ces caractéristiques ont une incidence directe sur les modalités d'accès à la terre au sein de ce groupe : la même enquête montre que cet accès s'est produit dans le cadre de la cellule familiale élargie ou d'une structure de type clanique (pour $73 \%$ des plus de 50 ans et $95 \%$ des cadets ayant moins de 35 ans), sous la forme d'un don ou d'un héritage ( $71 \%$ des plus de 50 ans et $93 \%$ des migrants les plus récents, pour une moyenne de $81 \%$ pour l'ensemble du groupe ; ibid. p. 171-2). Ces conditions permettent aux chefs de campement et, plus largement, aux chefs de famille installés sur le front pionnier de bénéficier d'apports réguliers en main-d'œuvre dont les services se perpétuent audelà de son accès au foncier, à travers des prestations exceptionnelles du type ukalè.

Les modalités d'insertion dans la société pionnière sont différentes dans le cas des Burkinabé mais elles tendent, avec le temps et le perfectionnement des stratégies de colonisation foncière, à reproduire, en les adaptant, les formes de l'organisation baoulé. Si la migration de colonisation des Burkinabé s'est réalisée avec un temps de décalage au regard de l'entreprise baoulé, c'est en grande partie du fait de l'absence d'une organisation clanique ou familiale qui soit à même d'en renforcer l'efficacité. Comme l'ont souligné différents auteurs [13-15], la migration burkinabé a d'abord été conçue comme une entreprise individuelle, hors d'un référent familial précis. Dès ses premiers développements dans le Sud-Ouest, elle s'est cependant appuyée sur un nombre limité 
d'intermédiaires, opérant au titre de l'ancienneté de leur installation dans les différentes zones d'accueil, qui ont constitué de véritables relais d'information, autant pour les migrants que pour les autorités administratives ivoiriennes ou burkinabé. Ces « chefs de communauté » ont ainsi joué le rôle de plaque tournante de la migration, désignant à chaque nouvel arrivant un tuteur originaire de sa petite région ou de sa sous-préfecture, qui lui fournirait gîte et nourriture ainsi qu'un appui dans la recherche de travail [14]. Se sont ainsi constituées des " chaînes migratoires " permettant une très forte mobilité spatiale, au sein desquelles chacun est appelé à assumer à son tour les fonctions d'accueil et d'assistance aux nouveaux arrivants.

Mais, parallèlement au fonctionnement de ces réseaux, l'installation d'un nombre croissant de planteurs burkinabé a fourni la base sociale d'un second type d'organisation, qui s'appuie éventuellement sur les services logistiques des premiers mais qui repose fondamentalement sur une structure de type familial ou villageois. Les vagues les plus récentes - et les plus denses - de migrants répondent en effet à l'appel des planteurs installés sur la frontière agricole, qui assument les frais de transport et canalisent les arrivées de leurs parents ou d'autres ressortissants de leur village d'origine. Les campements qui se développent sur le front pionnier tendent ainsi à regrouper des personnes originaires d'un même village ou d'une même petite région et sont l'expression de stratégies conçues dans un cadre familial.

Les données de l'enquête réalisée en 1994 dans la sous-préfecture de Méadji [13] mettent en évidence ce glissement graduel vers une organisation familiale tournée vers la colonisation : alors qu'à peine 17 $\%$ des planteurs burkinabé de plus de 50 ans sont arrivés sur le front pionnier par l'intermédiaire d'un parent, cette proportion s'élève à $42 \%$ chez les moins de 35 ans (30\% sur l'ensemble de l'échantillon). De même, les membres de la classe d'âge la plus élevée ont généralement accédé à la terre par I'intermédiaire d'un tuteur autochtone (75\% des cas), alors que cet accès a été organisé dans le cadre des réseaux burkinabé pour près de $55 \%$ des planteurs de moins de 35 ans.

Dans la mise en œuvre de ces stratégies, l'institution du " petit-frère " occupe une place centrale. Plus qu'un lien biologique, cette dénomination souligne la position de cadet social dans laquelle le nouvel arrivant est confiné, ainsi que les devoirs d'assistance auxquels il est soumis au-delà de son accès au foncier. Il peut demeurer à la disposition de son tuteur pendant plusieurs années, dans l'attente d'une rémunération différée sous forme de terre ou, plus rarement, d'un petit fonds de commerce ; durant cette période, il ne bénéficie que du minimum nécessaire à l'entretien de sa force de travail (hébergement, alimentation, soins de santé) mais en aucun cas d'un salaire ayant fait l'objet d'une convention, comme c'est le cas des " six-mois " baoulé. Les planteurs burkinabé bénéficient ainsi d'une main-d'œuvre semi-captive et très bon marché, dont le coût d'entretien est de surcroît limité par le type de nourriture qui constitue la base du régime alimentaire ${ }^{6}$, et dont le taux d'exploitation ${ }^{7}$ permet de dégager des profits qui seront partiellement réinvestis dans l'achat de terre ou dans d'autres activités économiques, en paiement différé du travail fourni. Les « petits-frères » constituent par ailleurs les forces vives des sociétés d'entraide, qui jouent un rôle important à l'occasion des pointes de travail du calendrier agricole (défrichements, sarclages, récolte et écabossage du cacao).

Les pratiques des Burkinabé en matière d'encadrement du processus migratoire, de constitution des cellules de colonisation du milieu et de régulation de l'accès au foncier se rapprochent donc du " modèle baoulé » de reconstruction d'une cellule familiale élargie, au même titre que leurs techniques de défrichement et de mise en place des cacaoyères tendent à reproduire celles que ce groupe a appliquées avec le succès que l'on sait. Elles s'en distinguent cependant par les facultés qu'elles 
confèrent aux planteurs burkinabé en termes d'exploitation de la main-d'œuvre et de « clientélisation » des cadets sociaux.

De façon transverse aux différents groupes impliqués dans le processus de colonisation, les institutions régulant la migration et l'accès aux ressources participent donc de la construction de rapports clientélistes se fondant sur les principes de réciprocité. Le développement des marchés de la terre et du travail débouche ainsi sur leur cloisonnement, sur la base des clientélismes internes aux différents groupes. Cette tendance, qui apparaît antagonique avec la dynamique de diffusion des relations marchandes, contribue en fait à sécuriser les activités productives, en simplifiant les cadres normatifs de référence et en réduisant les coûts de transaction. Comme les années 90 en fourniront la démonstration, la densité de ce type de rapports et l'existence de marchés cloisonnés constituent des amortisseurs de crises, que celles-ci soient d'origine externe (baisse des cours internationaux) ou interne (appauvrissement du milieu cultivé sous l'effet de la déforestation et renchérissement des coûts d'accès à la terre).

Des institutions évoluant sous lourde contrainte : la mobilité comme support de la reproduction sociale

Pour autant, la pérennisation des rapports clientélistes se heurte à des limites qui correspondent à celles, physiques, de l'espace foncier : d'une part, l'allégeance des cadets sociaux et le niveau de leurs transferts s'amenuisent avec le temps - à moins que leurs tuteurs ne puissent mettre en œuvre des mécanismes coercitifs à leur encontre, notamment en les coupant à leur tour des sources de maind'œuvre ; d'autre part, les possibilités d'élargir les clientèles sont limitées par les disponibilités foncières décroissantes - à moins d'effectuer un nouveau déplacement vers la frontière agricole. De façon générale, ces tendances requièrent une mobilisation croissante de capital de la part des maîtres de clientèles, qu'il s'agisse pour eux de maintenir ouvert leur accès au foncier ou de recruter leur maind'œuvre au prix fort, hors des allégeances clientélistes. Ces contraintes posent des limites au développement des exploitations et contredisent I'hypothèse d'une relation linéaire entre les acquisitions foncières et les surfaces effectivement mises en valeur par l'acquéreur. L'équivalence terre/travail, qui prend son expression dans le rapport entre la surface cédée dans la construction de clientèles et celle que ces dernières permettent de planter et d'entretenir, suit un rendement décroissant : le coût marginal de l'extension des exploitations cacaoyères ne cesse de s'accroître et exige des colons des cessions de terres - ou la mobilisation d'un capital extérieur - sans cesse plus importantes [8].

De façon plus générale, les processus de colonisation agraire qui se sont développés dans le Sud-Ouest ivoirien entre 1970 et 1990 ont débouché sur une monospécialisation culturale et une extrême fragilité structurelle des exploitations. Les logiques d'occupation rapide de l'espace et de " marquage » de l'appropriation foncière d'une part, et l'évolution des prix agricoles, qui renforçait la rentabilité de la culture cacaoyère au regard des autres productions, pérennes ou vivrières, d'autre part, ont conduit à la généralisation de techniques de culture qui associaient systématiquement la plantation de fèves de cacao aux défrichements et à la réalisation des cultures vivrières. De façon parallèle aux transformations observées dans l'organisation de la colonisation, ce phénomène correspond à l'adoption, par l'ensemble des groupes de migrants, des techniques baoulé de mise en valeur du milieu, qui se sont avérées les plus performantes au regard des objectifs d'appropriation foncière rapide et de maximisation de la productivité du travail : grâce à l'excellente complémentarité agronomique de l'igname, de la banane plantain et du cacao, les coûts de mise en place des vergers 
étaient pratiquement couverts par la production alimentaire et la commercialisation des excédents vivriers $[7,9]$.

L'effondrement de la filière de commercialisation du cacao, à la fin des années 80 , a montré à quel point cette monospécialisation fragilisait l'ensemble du tissu social de la frontière agraire. Cette fragilité est apparue d'autant plus forte que les exploitations pionnières doivent faire face à l'impossibilité de perpétuer l'association entre cultures vivrières et culture cacaoyère une fois leurs réserves forestières consommées et leur verger arrivé à maturité : elles dépendent alors du marché pour leur approvisionnement alimentaire - qu'il s'agisse d'y acquérir les denrées qu'elles ne peuvent plus produire ou de recourir à la location de friches pour poursuivre cette production ${ }^{8}$ - et doivent faire face à des coûts croissants, tant en termes monétaires que de travail fourni, pour atteindre ce but.

De façon générale, les exploitations pionnières ont à faire face à une baisse tendancielle de leurs performances économiques, ce que Ruf $[6,16]$ identifie à la dissolution d'une rente différentielle associée à la mise en valeur du milieu forestier originel. Il suffit pour notre propos de préciser ici que la destruction de ce milieu et le déplacement de la frontière agricole entraînent l'apparition de surcoûts (multiplication de la végétation adventice et des parasites du cacaoyer, baisse de la fertilité minérale et hydrique des sols ; accroissement du coût de la main-d'œuvre du fait de l'impossibilité de perpétuer l'échange terre-travail et de l'affaiblissement des allégeances clientélistes) qui se combinent au vieillissement des vergers et à la dissociation spatiale des cultures vivrières et pérennes pour provoquer une baisse sensible de la productivité du travail. Après la phase d'expansion et de marquage de l'appropriation foncière, extensive par nature, le renchérissement du travail freine ainsi le passage à des systèmes de production plus intensifs.

Ces blocages s'expriment de façon particulièrement aiguë à l'occasion du renouvellement des cacaoyères et expliquent l'échec de la plupart des tentatives de replantation. Ce phénomène n'est pas étranger à la superposition du cycle de vie biologique des plantations et du cycle domestique des planteurs qui, 25 à 30 ans après leur installation sur la frontière agricole et alors qu'ils doivent envisager le transfert de leur patrimoine à leur descendance, sont rarement en mesure de mobiliser le capital social et financier requis pour se lancer dans une entreprise ardue et coûteuse de replantation. D'une part, l'émergence de rentes foncières et les coûts sociaux d'élaboration et de validation des droits d'héritage se traduisent par un accroissement global des coûts d'accès au foncier pour leurs successeurs ${ }^{9}$. D'autre part, la mise en œuvre par les planteurs de stratégies d'extraversion (forte scolarisation des enfants préparant leur sortie de la sphère agricole, construction en ville ou dans le village d'origine) contribue souvent à limiter leur capacité d'investissement et d'entretien de leur capital productif, tout en hypothéquant leur contrôle ultérieur sur la main-d'œuvre familiale [10, 18].

Les dispositifs de régulation sociale et les systèmes de production agricole caractéristiques de la phase pionnière sont donc d'emblée confrontés à de lourdes contraintes, à la fois écologiques, agronomiques, économiques et sociales, qui encadrent étroitement les évolutions possibles de l'agriculture de plantation. Ces contraintes ont présidé au déplacement des bassins de production cacaoyère, depuis le Sud-Est du pays vers la " boucle du cacao " (départements de Bongouanou et de Dimbokro), puis le Centre-Ouest et le Sud-Ouest, en une succession de booms démographiques et économiques suivis de récessions tout aussi spectaculaires [6]. Tant que la frontière agricole est demeurée ouverte, et plutôt que d'assumer les faibles niveaux de rémunérations que procurent la prise en charge d'un verger sénescent ou une opération coûteuse et hasardeuse de replantation, les 
générations suivantes ont eu tendance à se déplacer vers de nouvelles terres forestières, porteuses de rente différentielle, et à entamer un nouveau cycle pionnier. La migration a ainsi constitué le mode de reproduction privilégié des exploitations cacaoyères. Cette logique a notamment conduit à l'émergence d'une classe de planteurs cumulateurs, généralement baoulé, qui ont suivi le déplacement géographique des foyers de production cacaoyère depuis le Centre-Est en direction du Sud-Ouest, jusqu'à butter sur la frontière libérienne $[9,19,20]$.

Ces stratégies de déplacement générationnel n'ont pas pour autant conduit à une reproduction à I'identique des structures d'exploitation, du fait notamment de la " massification » du processus migratoire mais aussi de la spécialisation croissante dans la production cacaoyère. L'enquête menée par le Cirad en 1993/1994 dans l'Est, le Centre-Ouest et le Sud-Ouest ([21] p. 96) met ainsi en évidence la réduction tendancielle de la surface des exploitations au fil de la dynamique pionnière (la moyenne passant de 19,8 à 17,6 et à 13,4 hectares) et, parallèlement, l'augmentation de la surface relative allouée à la culture cacaoyère $(61,75$ et $86 \%$ respectivement) ; selon la même enquête, une exploitation sur cinq dans le Sud-Ouest ne dispose d'aucune réserve foncière (ibid. p. 115). Les contraintes d'approvisionnement vivrier y conduisent ainsi à une dépendance structurelle croissante vis-à-vis des marchés (qu'il s'agisse des marchés des denrées alimentaires ou des marchés fonciers, pour la location de friches), qui s'est avérée particulièrement dramatique dès lors que la chute des prix du café et du cacao n'a plus permis d'en assumer les coûts.

\section{Crise du modèle pionnier et recomposition des dispositifs de contrôle social : l'« innovation-cannabis " en relais de la rente forestière}

Crise de l'agriculture pionnière : blocage de la mobilité sociale et remise en question des rapports de tutorat

Ces caractéristiques structurelles expliquent la brutalité de la crise qui a saisi l'ensemble de la société pionnière lors de l'effondrement des prix du cacao et du café entre 1989 et 1993. Son impact est largement perceptible à travers l'évolution des structures démographiques : dans des proportions variables suivant l'ancienneté des fronts de colonisation et les groupes sociaux, mais suivant un phénomène généralisé, les flux démographiques se sont inversés dans l'ensemble de la région SudOuest. Entre 1988 et 1994, les fronts pionniers de la rive ouest du Sassandra (sous-préfectures de Méadji et de Sassandra) ont ainsi vu leur population diminuer de 0,8 \% par an (après avoir connu une croissance annuelle de plus de $16 \%$ entre 1975 et 1988), reflet d'un déficit migratoire (- 4,2 \% par an) que l'accroissement naturel (+3,4\%) n'a pu compenser ([13] p. 203). Au total, ce sont $41 \%$ des résidents de 1988 qui étaient partis six ans plus tard, alors que seulement 17,6\% des personnes présentes en 1994 étaient arrivées au cours de ces six années de crise. Cette inversion des mouvements migratoires a touché les différentes catégories sociales de façon différenciée : ce sont surtout les cadets sociaux de sexe masculin, de 20 à 34 ans, qui travaillaient comme aides familiaux ou, plus fréquemment, comme salariés ou métayers, qui sont partis, permettant que se comble totalement le déséquilibre du sex-ratio chez les Baoulé et qu'il se réduise de près de $50 \%$ chez les Burkinabé (ibid.). Significativement, c'est au sein de ce dernier groupe, qui fournissait les plus gros contingents de main-d'œuvre, que l'émigration s'est avérée la plus forte (- 4,1 \% par an), même si elle a atteint un niveau également très élevé chez les Baoulé (- 3,2 \% par an sur la même période).

Cette évolution est directement liée au ralentissement considérable des processus de mobilité sociale sous l'effet conjugué de la crise économique et de l'épuisement de la frontière agricole. Sur les fronts pionniers de la rive ouest du Sassandra, on observe dans l'ensemble des groupes une augmentation 
sensible de l'âge au premier mariage : le taux de célibat des hommes de 20 à 34 ans est ainsi passé de 53 à $57 \%$ chez les Baoulé et de 50,5 à 62,5 \% chez les Burkinabé entre 1988 et 1994 ([13] p. 217-8) ; dans le même temps, la proportion de jeunes adultes chefs d'exploitation déclinait de façon uniforme : parmi les hommes de moins de 35 ans, elle est passée de 33 à $25 \%$ chez les Baoulé et de 34,5 à 17,8\% chez les Burkinabé ${ }^{10}$.

Cette moindre mobilité sociale ne se traduit pas pour autant par un contrôle accru de la part des chefs d'exploitation sur leur main-d'œuvre, comme l'atteste l'inversion des flux démographiques. On constate ainsi un repli marqué de l'organisation productive sur la famille restreinte, la parentèle élargie ou le village d'origine cessant de constituer les cadres de référence de l'approvisionnement en main-d'œuvre. La rapidité de cette évolution exclut par ailleurs l'éventualité d'une simple substitution de la force de travail provenant du groupe social élargi par la descendance biologique des planteurs : chez les Baoulé, les deux tiers des collatéraux (neveux, cousins, parents utérins) présents en 1988 avaient émigré six ans plus tard, leur part dans la population adulte totale diminuant de 30 à $16 \%$, alors que celle des enfants biologiques augmentait de 15 à $27 \%$ (ibid. p. 210-1) ${ }^{11}$. Au sein de la population burkinabé, ce sont les membres non apparentés du ménage qui ont fait les frais des ajustements consécutifs à la crise : leur représentation dans la population adulte a diminué de moitié au cours de cette période, en passant de 31 à $16 \%$. Cette évolution est due à l'émigration des neuf dixièmes des individus qui se trouvaient dans cette situation en 1988.

Ces ajustements affectant la main-d'œuvre non biologique se sont traduits par un accroissement global de la surface cultivée par actif et par la réduction des travaux d'entretien des plantations. Combinés à la réduction des charges d'exploitation (intrants, renouvellement du matériel, aménagements fonciers), ils ont permis d'enrayer sur le court terme l'effondrement des niveaux de productivité individuelle, mais ont également eu pour corollaire une dégradation sensible de l'état phytosanitaire des vergers et l'accélération des processus de vieillissement végétatif de ces derniers, réduisant leur potentiel de production. Sur l'ensemble de l'échantillon enquêté, les rendements cacaoyers ont ainsi subi une régression variant de 15 à $20 \%$, dans les systèmes d'exploitation les plus intensifs, à plus de $30 \%$ dans les systèmes extensifs en travail [21]. Cette érosion des rendements physiques et des revenus monétaires a souvent été suivie d'un nouvel allègement des charges d'exploitation : bon nombre d'unités de production se sont ainsi engagées dans un processus cumulatif d'« extensification » (réduction des entretiens - diminution des rendements - etc.) qui a débouché sur l'abandon des plantations les moins productives ${ }^{12}$.

Ces deux tendances lourdes, blocage de la mobilité sociale et décapitalisation plus ou moins rapide portant sur le patrimoine-plantation encadrent étroitement les dynamiques institutionnelles. Elles renvoient, en fait, à une remise en question des rapports de tutorat ayant régi les processus d'accès à la terre et au travail, via les institutions du " petits-frères " et du " six-mois », en particulier au niveau des exploitations de taille moyenne ou petite qui ne disposent pas d'une surface financière suffisante pour pourvoir aux coûts salariaux, de transport, d'alimentation et de santé des travailleurs et, surtout, ne peuvent plus leur offrir la perspective d'une rémunération différée sous la forme d'un accès aux terres forestières. Avec cette remise en cause des piliers institutionnels qui organisaient l'accès aux ressources et fondaient les mécanismes d'intégration sociale, c'est l'organisation même de la société pionnière et les compromis entre ses différentes composantes qui sont menacés (pérennité des allégeances clientélistes, stabilité des relations intergénérationnelles et intercommunautaires, organisation interne aux différentes diasporas). 
La précarisation de la position des cadets sociaux va ainsi de pair, de façon apparemment paradoxale, avec l'affaiblissement de l'autorité des chefs de famille et des chefs de communauté, dès lors que le cadre institutionnel sur lequel reposait leur pouvoir s'avère insuffisant pour garantir la régulation sociale. II résulte de la conjonction de ces facteurs une fragmentation de l'autorité au sein des groupes domestiques comme des différentes communautés et une forte diversité des normes régissant les rapports entre individus : la mise en œuvre par les cadets de stratégies d'affiliation à d'autres groupes domestiques, en fonction de leur assise foncière, et la diffusion de pratiques clientélistes, parallèlement aux relations de patronage qui marquaient les rapports entre le migrant et l'aîné de la migration ou entre celui-ci et son bailleur de terre, sont les principales expressions de ce phénomène.

\section{Un facteur de stabilisation des institutions de la frontière : le trafic de marihuana}

L'épuisement de la frontière agricole et la crise cacaoyère ont encouragé, depuis la fin des années 80 , l'expression de multiples initiatives paysannes en matière de développement de nouvelles activités productives. Celles-ci ont concerné principalement des productions à valeur pondérale élevée, qui permettent de surmonter les coûts de transaction et d'évacuation inhérents à la mise sur le marché dans les situations d'enclavement qui caractérisent les anciennes zones de frontière. On peut citer, pêle-mêle, le développement des productions maraîchères, des petits élevages et, plus spécifiquement, de la pisciculture. La production de cannabis semble cependant promise au plus large succès dans la mesure où elle offre, de façon paradoxale s'agissant d'une activité illégale, une plus large sécurité en matière de maîtrise technique et de mise sur le marché.

Bien qu'elle ait localement fait l'objet d'un usage domestique dès les années 50 , au contact forêtsavane ou dans la zone littorale, la culture du cannabis n'a réellement diffusé en zone forestière ivoirienne qu'à la fin des années 80. Parmi les producteurs de marihuana enquêtés sur les fronts pionniers des sous-préfectures de Méadji et Sassandra, la quasi-totalité ( $92 \%$ ) a adopté cette culture après l'effondrement des prix du café et du cacao en 1988/1989 ${ }^{13}$. II s'agit d'un phénomène aussi généralisé que rapide puisque, au-delà de la diversité socioculturelle de la population, presque tous les groupes apparaissent impliqués, à des degrés divers, dans ce trafic. La recherche de revenus alternatifs à ceux du cacao et la nécessité pour de nombreux jeunes d'assurer leur avenir sur des surfaces réduites et des sols appauvris apparaissent ainsi comme les principaux moteurs de sa diffusion. Son essor ne peut toutefois être dissocié de l'accroissement de la demande locale : au plus fort de la crise, la consommation de stupéfiants, amphétamines en premier lieu mais aussi marihuana, a été adoptée par certains comme un moyen de compenser les départs de travailleurs salariés en augmentant la durée quotidienne du travail ${ }^{14}$.

L'organisation sociale et foncière propre à chaque communauté ainsi que les réseaux marchands qui s'étaient constitués sur la frontière agricole ont fourni un support efficace à cette diffusion. D'une part, l'individualisation du foncier et l'affaiblissement des allégeances clientélistes au fur et à mesure du vieillissement de la société pionnière ont érodé les formes de contrôle social. D'autre part, le trafic a pu se greffer sur un réseau dense de commerçants et de transporteurs mis à mal par la crise du secteur café-cacao : de par les volumes considérables échangés et la fréquence des convoyages, il a constitué un support idéal pour le commerce du cannabis et représentait au milieu des années 90 le principal canal de mise sur le marché de la production (voir infra).

L'évolution du couvert végétal au profit des cacaoyères et des jachères à Chromolaena odorata a enfin constitué un facteur de poids dans le développement des cultures illicites : $C$. odorata tend à former un fourré dense et impénétrable qui dissimule parfaitement les cultures aux regards indiscrets ; une 
trouée effectuée dans une plantation cacaoyère à plus d'une trentaine de mètres d'un chemin est de même pratiquement invisible. Près des deux tiers des producteurs interrogés (62\%) pratiquaient la culture du cannabis dans des clairières ménagées au sein des cacaoyères, $33 \%$ au milieu d'une friche de $C$. odorata ou d'un champ de manioc, lui-même établi derrière un paravent d'adventices. Du fait de sa très forte valeur pondérale, le cannabis est par ailleurs cultivé sur de très petites surfaces : $30 \%$ des producteurs enquêtés semaient moins de 10 ares par an et les deux tiers le cultivaient sur moins d'un quart d'hectare. En dépit de l'accroissement des densités de population, la réduction des surfaces à la disposition des jeunes paysans n'a donc pas constitué un obstacle au développement des cultures clandestines.

Le trafic de marihuana s'est ainsi inséré dans les stratégies de diversification ou de reconversion développées par l'ensemble des acteurs de la filière cacao. Ce contexte particulier ainsi que la " rente de situation " dont bénéficie la Côte d'Ivoire dans ses relations avec les pays occidentaux et les bailleurs de fonds peuvent expliquer la faiblesse de la répression au moment de l'enquête (1995). La petite paysannerie bénéficiait alors d'un accès relativement aisé au trafic, qui contrastait avec les structures coercitives dominant cette activité en Amérique latine, par exemple.

La culture du cannabis concerne ainsi majoritairement des personnes dont l'accès au foncier est apparemment bloqué : la moitié des producteurs de marihuana enquêtés pratiquaient cette culture en tant que contractuels sur l'exploitation d'un proche, parent, membre de leur clan ou originaire de la même petite région (figure 1). Ils ignoraient qui en était le commanditaire et quels en étaient les profits réels, mais bénéficiaient d'une bonne protection vis-à-vis des forces de l'ordre. Leur rémunération était souvent différée pendant plusieurs saisons, jusqu'à ce que leur employeur leur cède un lopin de forêt, une plantation, un petit commerce ou un taxi collectif, en une sorte de reproduction des modalités de fixation et de rémunération de la main-d'œuvre propres au rapport « petit frère/grand-frère " instauré durant la phase pionnière. Dans les cas où ces manœuvres étaient rémunérés de façon régulière, leurs revenus correspondaient à une norme relativement uniforme, attribuant une valeur de 300000 à 350000 FCFA à la culture de 625 à $1000 \mathrm{~m}^{2}$ (I'unité de référence étant un carré dont les côtés varient de 25 à $35 \mathrm{~m}$ ), pour chacun des deux à trois cycles de culture pratiqués dans l'année.

L'autre moitié des producteurs de marihuana était majoritairement composée de paysans ayant un accès limité à la terre, parfois même à une petite plantation, mais qui se trouvaient en situation de blocage foncier : $20 \%$ d'entre eux n'avaient pas de plantation et la moitié disposait d'un verger de moins de 3 hectares. Certains, dont l'assise sociale au sein du village était particulièrement précaire, s'étaient placés sous la protection d'un gros producteur de marihuana, dans des conditions de subordination similaires à celles qui prévalaient pour les paysans sans terre. La plupart bénéficiait cependant d'un accès plus favorable au marché : leur récolte n'était pas vendue au forfait mais en fonction de son volume, le sac utilisé pour la vente du cacao constituant l'unité de mesure. L'association avec un intermédiaire restait toutefois de rigueur afin de s'assurer de la commercialisation rapide et complète de la récolte et pour bénéficier d'une protection sûre. Comme dans le cas précédent, le commanditaire fournissait les semences et garantissait le prix d'achat. La valeur bord-champ d'un sac rempli de 20 à $25 \mathrm{~kg}$ de feuilles séchées s'établissait autour de 150000 à 200000 FCFA et le produit brut obtenu sur 0,1 hectare, équivalant à la production de 8 à 12 sacs, variait entre 1,5 et 2 millions de FCFA.

Quelques producteurs (environ $15 \%$ des personnes interrogées) vendaient au détail une partie de leur 
récolte sur les marchés de proximité qui se sont développés avec la crise. Ces paysans étaient amenés à prendre en charge les fonctions assumées par les commanditaires dans les cas précédents, en particulier l'achat de protections qui pouvait absorber une grosse partie de leur revenu ${ }^{15}$. En fait, la récolte était rarement vendue au détail dans sa totalité, mais plutôt à des " dealers " locaux ou à des boutiquiers qui remplissaient cette fonction dans les différents villages. Un sac de $20-25 \mathrm{~kg}$ de marihuana leur rapportait en moyenne 500000 FCFA, et la culture de 0,1 hectare entre 3 et 5 millions de FCFA.

De tels niveaux de rémunération sont bien sûr de nature à bouleverser les conditions de reproduction de la société agraire : en 1995, il suffisait de vendre un sac et demi de marihuana, soit la production de $150 \mathrm{~m}^{2}$ environ, pour couvrir les besoins de base d'une famille (figure 2) ; la production de 0,1 ha vendue " au sac " procurait, sur deux cycles de culture, un revenu annuel de 3 à 4 millions de FCFA, soit la valeur de 10 à $13 \mathrm{t}$ de cacao, le produit d'une plantation d'une trentaine d'hectares exploitée en faire-valoir direct, grâce à une dizaine de travailleurs, ou de plus de 40 ha confiés en métayage. Les revenus annuels générés par le cannabis se situaient en moyenne à 4500000 FCFA (de $700000 \mathrm{~F}$ pour les producteurs rémunérés par unité de surface à 9000000 FCFA si le chanvre était vendu au détail), soit 11 à 12 fois la valeur des besoins de base d'une famille entière [24].

L'impact potentiel du cannabis est d'autant plus fort que cette culture s'insère dans les systèmes de production sans concurrencer les autres activités agricoles, ni dans l'occupation de l'espace, ni dans l'organisation du travail. Les cultures licites, qu'il s'agisse du café et du cacao ou des productions vivrières, demeurent des façades indispensables pour justifier un enrichissement vis-à-vis de la communauté villageoise. En raison des petites surfaces occupées par les plantations de cannabis et de la nécessité de maintenir une discrétion absolue, les travaux y sont par ailleurs réalisés en fin de journée, lorsque les propriétaires des parcelles voisines sont déjà retournés au village. Le coût d'opportunité du travail consacré aux cultures illicites est donc très faible et ne pénalise pas les autres productions.

Les synergies apparaissent particulièrement fortes entre le cannabis et la culture cacaoyère. Alors que le renouvellement des vergers cacaoyers exige une injection de capital et de travail que peu de cadets sociaux sont en mesure de fournir, la plantation (ou la replantation) procure en retour une excellente valorisation des revenus illicites, dans la mesure où elle fournit une base foncière et un paravent économique nécessaires à la poursuite de cette activité. Ainsi, même si les paysans interrogés orientaient plutôt leurs investissements vers le secteur tertiaire (transport, immobilier, commerce), notamment parce qu'il leur apparaissait plus facile de les rentabiliser à distance de la société villageoise, près du tiers d'entre eux avait acquis une plantation ou un îlot de forêt grâce aux revenus du cannabis.

Plus largement, la production de marihuana apparaît soutenir une économie régionale gravement affectée par la crise. Tous les producteurs interrogés avaient ainsi réinvesti une grande partie de leurs profits dans le tissu économique de la région : dans l'immobilier urbain (immeubles de rapport et hôtellerie) pour 32 \% d'entre eux, le commerce (épiceries, collecte de café et de cacao, vente en gros de riz) dans $29 \%$ des cas et les transports (taxis urbains et taxis-brousse, véhicules de collecte du cacao) pour $45 \%{ }^{16}$. Si bon nombre de ces investissements visaient la création de rentes immobilières, une part significative participait du développement économique, comme la construction ou le rachat d'hôtels, l'acquisition de taxis-brousse, l'achat de plantations ou l'installation de commerces ayant pour objet l'achat et la vente de produits agricoles. La fréquence des investissements dans le 
commerce de cacao ou de café n'est pas fortuite : si l'on excepte la distribution de marihuana et d'amphétamines à l'intention des manœuvres et des petits paysans de la région, les principaux réseaux de portée nationale s'identifient avec les circuits de commerce du café et du cacao ${ }^{17}$ [24].

Un intermédiaire d'un grand centre de transit routier faisait ainsi état, en 1995, d'un trafic de 250 à $300 \mathrm{t}$ réalisé sous couvert des circuits d'évacuation du cacao vers le port d'Abidjan au cours des huit mois de traite. Si l'on se réfère aux quantités produites au niveau de l'échantillon enquêté, cette production correspondait à l'activité de 350 ou 400 paysans. Aux prix pratiqués dans le Sud-Ouest ivoirien, elle représentait un revenu brut de 2 à 2,5 milliards de FCFA, soit la valeur « bord-champ » de 6500 à 8000 t de cacao, $4 \%$ environ de la production du Sud-Ouest. Mais, sur les principaux marchés urbains du pays, la même quantité de marihuana représentait un revenu de 25 milliards, équivalant à la valeur bord-champ de la moitié de la production régionale de café et de cacao.

Le cas du trafic de cannabis nous fournit matière à réflexion quant aux conditions de diffusion des innovations techniques et institutionnelles dans le contexte particulier de la " frontière interne ». Tout se passe comme si l'innovation technique - l'introduction d'une nouvelle culture dans les systèmes de production et d'un nouveau produit dans les systèmes d'échange - permettait de différer ou de contourner les innovations sociales et institutionnelles que l'achèvement du cycle pionnier semblait imposer dans le champ de l'organisation économique. La diffusion de l'« innovation cannabis " s'effectue ici par le biais des institutions qui s'étaient mises en place au cours de la phase pionnière pour encadrer l'activité des migrants et réguler leur accès au foncier : le rapport de patronage liant le « petit-frère " à un tuteur déjà établi et/ou le contrat de métayage abu-san, qui accompagne ou prolonge fréquemment un premier contrat de type "six-mois", sont généralement les supports de productions illicites réalisées sous couvert de l'exploitation cacaoyère [26]. La culture du cannabis permet ainsi de perpétuer les mécanismes de contrôle de la force de travail et les rapports de production liés à l'échange terre/travail au-delà de l'épuisement de leur support principal, la forêt. Audelà de la production elle-même, et grâce aux niveaux de rémunération élevés que procure la production de marihuana, les institutions correspondant aux différentes formes de tutorat entre planteurs et immigrants continuent à remplir leur rôle dans les dispositifs de régulation et de mobilité sociale.

De par sa nature illégale, soumise à répression, la production de cannabis contribue à renforcer, ou à rétablir, les mécanismes de régulation et d'intégration sociale, dont les groupes qui dominent le jeu local et domestique - les aînés sociaux, les détenteurs de terre - sont les principaux garants et bénéficiaires. Parce qu'elle est clandestine et que son accès se trouve conditionné à une sujétion plus ou moins forte aux structures locales et domestiques de pouvoir, elle ne peut induire une transformation radicale de l'organisation et des institutions à travers lesquelles s'exerce la régulation sociale (ibid.). De ce point de vue, la culture du cannabis complète le dispositif de densification des rapports clientélistes au sein de la société pionnière. Les relations de clientèle entre groupes, ou au sein de chaque groupe, constituent un support essentiel des dynamiques de diffusion des innovations, en même temps que ces dernières permettent la densification des rapports clientélistes.

Pour autant, l'organisation de la production illicite autour des institutions qui ont régulé l'échange terre/travail au cours de la phase pionnière ne remet pas en question l'instrumentation potentielle de ce rapport au bénéfice des travailleurs. Les stratégies d'insertion dans la sphère illégale se définissent au niveau individuel, peuvent s'établir dans le court terme et débouchent sur une autonomisation économique du petit producteur. Le contrôle des réseaux clandestins d'encadrement de l'activité 
illégale est réel, comme le sont les rapports de patronage qui lient le petit producteur au propriétaire ou au commanditaire, mais ils semblent avoir une prise limitée au-delà du cycle productif. Ces caractéristiques autorisent l'expression de stratégies temporaires de capitalisation dans la sphère illicite, puis de réalisation de ce capital et de réinsertion dans l'économie légale qui contrastent avec l'emprise que les structures mafieuses du trafic exercent sur leurs membres en Amérique latine, par exemple [27].

Cycles du cacao, logiques de l'État et innovations paysannes : accompagner les dynamiques locales, jusqu'où ?

Dans le développement de la production et du trafic de marihuana comme en bien d'autres matières, la Côte d'Ivoire et le Ghana semblent avoir suivi des trajectoires parallèles. Les similitudes dans les cycles cacaoyers qui ont marqué l'histoire économique et politique des deux pays ainsi que le décalage entre ces deux cycles, du fait principalement des politiques divergentes adoptées par les anciennes tutelles coloniales vis-à-vis du secteur indigène de l'agriculture, ont été amplement soulignés par différents auteurs $[6,28,29]$. Les similitudes des réponses politiques apportées aux crises induites par l'achèvement de ces cycles cacaoyers, en dépit de leur décalage et des leçons historiques de l'expérience ghanéenne, n'en sont pas moins frappantes - de l'accroissement des ponctions étatiques sur le secteur paysan au durcissement de la politique migratoire et la précarisation du statut des producteurs étrangers, en passant par l'intervention des militaires dans l'arène politique nationale. Un autre élément de rapprochement des deux trajectoires nationales réside dans l'essor du trafic de cannabis et la concentration de sa production sur les anciens fronts pionniers cacaoyers $[24,25]$. Tout se passe comme si les activités illicites s'inscrivaient de façon " naturelle » dans le prolongement des cycles de développement, d'apogée et de déclin des économies de plantations ouest-africaines [30].

L'« innovation cannabis " apparaît comme la plus pertinente - et à certains égards comme la plus accessible - alternative de reconversion à l'issue du cycle cacaoyer pionnier, non seulement pour les exploitations paysannes mais aussi pour les réseaux marchands et l'ensemble du système de régulation politique qui, jusqu'alors, avait reposé sur la gestion centralisée des rentes tirées des exportations de café et de cacao. Dans une certaine mesure, sa diffusion peut être comparée à celle de l'« innovation cacaoyère " à la fin du xix siècle au sein des sociétés yoruba au Nigeria, akwapim et krobu au Ghana, dont les bases économiques avaient été affectées par la dépression du marché de I'huile de palme puis par la colonisation européenne [28]. II est remarquable que, dans le cas du cannabis, cette diffusion se produise apparemment sans induire de transformation majeure de I'organisation économique et sociale. En Côte d'Ivoire, elle permet au contraire, à ce stade de son développement, la reproduction des institutions qui ont structuré les relations entre les différentes composantes de la société agraire et du secteur cacaoyer, et apparaît comme un support possible des filières exportatrices.

Eu égard au contrôle exercé par l'État ivoirien et ses représentants sur ces filières, il est difficile d'envisager que le trafic de marihuana ait pu s'appuyer sur ces dernières sans l'implication, au moins passive, des élites politico-administratives. Au-delà des possibilités d'enrichissement qu'il offre aux plus hauts responsables, le trafic de drogues apparaît plus largement comme un facteur potentiel de stabilisation des dispositifs de contrôle sociopolitique au niveau de groupes sociaux ou de portions du territoire national que la crise menace de déstabiliser. L'érosion des rentes à l'exportation s'étant traduite, dans les deux pays, par de grandes difficultés à maintenir les niveaux de financement des clientèles politiques et des pouvoirs locaux, le trafic de drogue est susceptible de combler localement 
les déficiences du pouvoir central, moyennant la délégation à ces pouvoirs des responsabilités financières qu'il assumait auparavant.

Le maintien de la légitimité de l'État et de ses représentants dépend alors de leur capacité à imposer une certaine régulation aux activités illicites. La répression de la production et du trafic ainsi que ses modalités de mise en œuvre doivent être analysées en relation à cette contrainte. Le contrôle des grandes filières d'exportation, par lesquelles semblait transiter la plus grande partie du cannabis destiné au marché international au milieu des années 90 , demeurant alors entre les mains des élites politiques, le narcotrafic ne paraissait pas remettre en question le système centralisé de gestion des rentes et de financement des clientèles : la répression vis-à-vis des producteurs de cannabis était particulièrement laxiste et semblait répondre davantage aux logiques d'autofinancement des appareils policier et judiciaire qu'à une volonté politique. À terme, l'émergence et la consolidation de pouvoirs locaux et régionaux s'appuyant sur une base économique et sociale très large est cependant susceptible de provoquer une remise en question de la légitimité de l'État. De régulateur, le pouvoir central devient un instrument au service des trafiquants. Une telle évolution semble déjà être en cours dans certains pays d'Amérique latine : le suivi attentif de la situation dans des pays comme le Ghana ou le Nigeria permettra de dire si un tel schéma est transposable à l'Afrique subsaharienne.

* Communication à la conférence internationale "L'avenir des cultures pérennes ", Bnetd-Cirad, 5-9 novembre 2001, Yamoussoukro, Côte d'Ivoire.

Notes :

${ }^{1}$ Sans que les informations disponibles permettent de préjuger de l'implication des principales firmes exportatrices de café et de cacao, qui ont par ailleurs constitué un élément clé du dispositif de structuration du système politique national, via le contôle de ces entreprises stratégiques par les élites composant le premier cercle du pouvoir houphouëtien.

${ }^{2}$ La loi foncière de 1962, qui tendait à renforcer les prérogatives de l'État sur le domaine foncier, ne sera jamais votée devant l'inconformité des autorités coutumières et des représentants politiques qui leur étaient liés.

${ }^{3}$ Le métayage sur les caféières ou sur des cacaoyères vieillissantes, que le travailleur est chargé de remettre en état, donne généralement lieu à un partage par moitié (abu-ñon) ; il s'agit d'une situation relativement marginale sur les fronts pionniers du Sud-Ouest.

${ }^{4}$ Ruf ([7] T, II : 45) évalue le coût moyen d'un « six-mois » à $469 \mathrm{FCFA} / \mathrm{j}$, contre $603 \mathrm{~F}$ pour un métayer abu-san et $1187 \mathrm{~F}$ dans le cas d'un travailleur rémunéré à la tâche.

${ }^{5}$ Le nombre et l'importance des filles " six-mois ", employées aux travaux domestiques, aux cultures vivrières et au transport des récoltes, est souvent sousestimé. Oswald ([11] p. 121) fait état d'une proportion pouvant atteindre le tiers de cette main-d'œuvre. Après une première expérience probatoire, il n'est pas rare que les jeunes hommes se voient proposer un contrat de partage de la récolte en abu-san, toujours sur la base de séjours de six mois.

${ }^{6}$ Au regard de son prix, le maïs offre en effet un rendement énergétique sans rapport avec ceux que présentent le riz ou l'igname, base alimentaire des groupes autochtones et baoulé, respectivement. 
${ }^{7}$ À un stade précoce du développement du front pionnier de Méadji et de la colonisation burkinabé, Léna [9] faisait déjà état de la durée très importante du travail quotidien au sein de ce groupe, phénomène qu'il reliait à la faiblesse du temps accordé à la vie sociale au sein des campements mossi.

8 Ruf [16] ajoute à ce coût croissant de l'approvisionnement vivrier celui qui résulte de l'appauvrissement de la faune sauvage, qui a fourni, via la chasse, une part importante de la diète alimentaire au cours de la phase pionnière.

${ }^{9}$ La transmission des plantations par filiation directe est fréquemment remise en cause au sein de la famille élargie, au profit d'une réactivation des normes traditionnelles opérant en faveur des aînés de lignage (dans le cas des autochtones kru) ou des parents utérins (chez les allochtones d'origine akan (voir Chauveau [17]).

${ }^{10}$ Notons par ailleurs que l'accès au statut de chef d'exploitation est souvent insuffisant pour acquérir une autonomie sociale : le nombre de planteurs est ainsi inférieur à celui des chefs de ménage dans le groupe d'âge des 20-34 ans, ce qui montre que l'accès à la terre ne suffit plus pour s'affranchir de la tutelle des aînés (Balac [13] p. 220).

${ }^{11}$ Sur l'ensemble du territoire de Sassandra, Guillaume et Vimard ([22] p. 328) observent sur la même période une diminution de moitié de la proportion d'enfants " non apparentés " hébergés par les familles baoulé.

${ }^{12}$ Les enquêtes réalisées en 1994 sur les fronts pionniers de la rive gauche du Sassandra montrent que $10 \%$ de la surface cacaoyère avait été ainsi « mise en sommeil » par $11 \%$ des planteurs ; dans le cas du café, ces proportions atteignaient $12,5 \%$ des vergers et $11,5 \%$ des exploitations.

${ }^{13}$ Les résultats présentés ici proviennent d'une enquête menée en 1994 et 1995 auprès de 41 producteurs de marihuana de la région Sud-Ouest et ont été exposés en détail dans deux travaux antérieurs (Léonard [23, 24]).

${ }^{14}$ Contrairement à la situation qui prévaut dans les pays occidentaux, où la consommation de cannabis répond essentiellement à un usage récréatif, son emploi en Afrique de l'Ouest semble associé à des situations perçues comme éprouvantes, voire dégradantes : travaux pénibles, activités moralement réprouvées (voir Laniel [25]).

${ }^{15}$ Selon le témoignage d'un producteur, ces frais représenteraient jusqu'à $50 \%$ du produit brut.

${ }^{16}$ Même si ce genre d'inventaire est à considérer avec précaution, les investissements déclarés par les 41 planteurs enquêtés sont éloquents : 2 hôtels, 24 immeubles de rapport (allant de la maison individuelle à la " cour » rassemblant plusieurs habitations), 4 terrains à bâtir, 6 taxis collectifs (18 à 22 places), 13 taxis urbains, 10 véhicules pour la collecte de cacao ( 3 à $5 \mathrm{t}$ de charge), 9 commerces fixes (de la boutique villageoise au magasin de vente de riz en gros), 11 plantations et 2 parcelles de forêt.

${ }^{17}$ Des quantités moins importantes de cannabis sont toutefois envoyées sur Abidjan dans les soutes des autobus de deux compagnies régionales, ou dans les ballots de cola fraîche expédiés vers le Mali et le Burkina Faso : s'agissant d'une denrée très valorisée sur les plans social et culturel, les chargements de cola sont rarement inspectés aux points de contrôle routier, car l'exposition de la cola à l'air provoquerait son oxydation et une dépréciation de la valeur marchande du produit. 


\section{REFERENCES}

1. KOPYTOFF I (1987). The internal African frontier: the making of african political culture. In : Kopytoff I, ed. The African Frontier. The reproduction of traditional African societies. Bloomington and Indianapolis : Indiana University Press : 3-84.

2. CHAUVEAU JP (2000). Question foncière et construction nationale en Côte d'Ivoire. Les enjeux silencieux d'un coup d'État. Politique Africaine, 78 : 94-125.

3. CHAUVEAU JP, DOZON JP (1985). Colonisation, économie de plantation et société civile en Côte d'Ivoire. Cahiers ORSTOM Sciences Humaines, XXI : 63-80.

4. CONTAMIN B, MEMEL-FOTE H (1997). Le modèle ivoirien en questions. Paris : Karthala/Orstom.

5. LOSCH B, éd. (2000). Côte d'Ivoire : la tentation ethno-nationaliste. Politique Africaine, 78.

6. RUF F (1995). Booms et crises du cacao. Les vertiges de l'or brun. Paris : ministère de la Coopération/Cirad/Karthala, $459 \mathrm{p}$.

7. RUF F (1988). Stratification sociale en économie de plantation ivoirienne. Thèse de troisième cycle de I'Université de Paris-X, 6 tomes ; 976 p., multigr.

8. LÉONARD E (1997). Crise écologique, crise économique, crise d'un modèle d'exploitation agricole. Ajustements et recomposition sociale sur les anciens fronts pionniers ivoiriens. In : Contamin B, Memel-Fotê $\mathrm{H}$, eds. Le modèle ivoirien en questions. Crises ajustements, recompositions. Paris : Karthala/Orstom : 393-413.

9. LÉNA P (1979). Transformation de l'espace rural dans le front pionnier du Sud-Ouest ivoirien. Thèse de troisième cycle de l'Université de Paris-X ; 345 p., multigr.

10. LESOURD M (1988). La forêt, la machette et le billet de banque. Problématique du colonat agricole en région d'économie de plantation : l'exemple du système de production baule (Côte d'Ivoire forestière). Cahiers ORSTOM Sciences Humaines, 24 : 78-97.

11. OSWALD M (1998). Recomposition d'une société au travers de plusieurs crises : la société rurale bété (Côte d'Ivoire). Thèse de doctorat de l'Ina-GP ; 300 p., multigr.

12. LESOURD M (1982). L'émigration baoulé vers le Sud-Ouest de la Côte d'Ivoire. Thèse de troisième cycle de l'Université de Paris-X, 2 tomes ; 526 p., multigr.

13. BALAC R (1998). Gens de terre, gens de réseaux : mécanismes de production et lien social. Thèse de doctorat de l'Institut d'Études Politiques de Paris ; 276 p., multigr.

14. BLION R, BREDELOUP S (1997). La Côte d'Ivoire dans les stratégies migratoires des Burkinabé et des Sénégalais. In : Contamin $\mathrm{B}$, Memel-Fotê $\mathrm{H}$, eds. Le modèle ivoirien en questions. Paris : Karthala/Orstom : 707-37.

15. BOUTILLIER JL, QUESNEL A, VAUGELADE J (1977). Systèmes socio-économiques mossi et migrations, Cahiers ORSTOM Sciences Humaines, 4 : 361-81. 
16. RUF F (1987). Éléments pour une théorie sur l'agriculture des régions tropicales humides. I. De la forêt, rente différentielle, au cacaoyer, capital travail. L'Agronomie Tropicale, 42 : 218-32.

17. CHAUVEAU JP (1997). Jeu foncier, institutions d'accès à la ressource et usage de la ressource. Une étude de cas dans le Centre-Ouest ivoirien. In : Contamin B, Memel-Fotê $\mathrm{H}$, eds. Le modèle ivoirien en questions. Paris : Karthala/Orstom : 325-60.

18. CHALÉARD JL (1993). Cocoa planters and land investment in towns in Côte d'Ivoire. Communication à I'International Conference on Cocoa Economy, Askindo-Cirad, Bali, 19-22 octobre 1993, 12 p., multigr.

19. AFFOU YS, TANO K (1992). Migration rurale et dynamique socio-économique. De la Boucle du Cacao au Sud-Ouest ivoirien. Abidjan : Cires-Aisa-Orstom ; 36 p., multigr.

20. BALAC R (1997). Peuplement et économie de plantation dans le territoire de Sassandra. In : Guillaume A, et al., eds. Croissance démographique, développement agricole et environnement à Sassandra. Orstom-Ensea-Gidis-Cl : 87-113.

21. LÉONARD E (1983). Différenciation et reproduction des exploitations agricoles dans le Sud-Ouest ivoirien : une typologie des systèmes de production. Document de travail, IRD, Abidjan, centre du Petit-Bassam ; 57 p., multigr.

22. GUILLAUME A, VIMARD P (1997). La circulation et l'activité des enfants de Sassandra. In : Guillaume $\mathrm{A}$, et al., eds. Croissance démographique, développement agricole et environnement à Sassandra. Paris : Orstom-Ensea-Gidis-Cl : 323-45.

23. LÉONARD E (1995). Développement de la culture du cannabis et du trafic de marihuana en Côte d'Ivoire forestière. Paris : Orstom/Observatoire géopolitique des drogues ; 46 p., multigr.

24. LÉONARD E (1998). Crises des économies de plantation et trafic de drogues en Afrique de l'Ouest. Les cas ivoirien et ghanéen. Autrepart, 8 : 79-99.

25. LANIEL L (1995). Développement de la culture du cannabis et du trafic de marihuana au Ghana. Paris : Observatoire géopolitique des drogues ; 39 p., multigr.

26. LÉONARD E (2001). Essor des cultures illicites et dynamique des institutions agraires dans les agricultures périphériques du Sud. Une comparaison Mexique-Côte d'Ivoire. In : Winter G, éd. Inégalités et politiques publiques en Afrique. Pluralité des normes et jeux d'acteurs. Paris : Karthala/IRD : 209-23.

27. LÉONARD E (1997). Crise et développement des cultures illicites au Mexique et en Côte d'Ivoire. In : Gastellu JM, Marchal JY, éds. La ruralité dans les pays du Sud à la fin $d u$ xx ${ }^{e}$ siècle. Paris : Orstom, coll. Colloques et Séminaires ; 301-31.

28 CHAUVEAU JP (1993). L'innovation cacaoyère : stratégies d'acteurs, institutions et contextes locaux. La diffusion comparée de la cacaoculture en Afrique de l'Ouest. In : L'innovation en milieu rural. Montpellier : Orstom : 135-65.

29. GASTELLU JM (1982). Les plantations de cacao au Ghana. Cahiers ORSTOM Sciences Humaines, 18 : 225-54. 
30. Observatoire géopolitique des drogues (1996). Étude régionale sur le trafic, la production et la consommation des drogues dans les pays d'Afrique de l'Ouest. Bruxelles : Commission des communautés européennes ; 193 p., multigr.

Illustrations

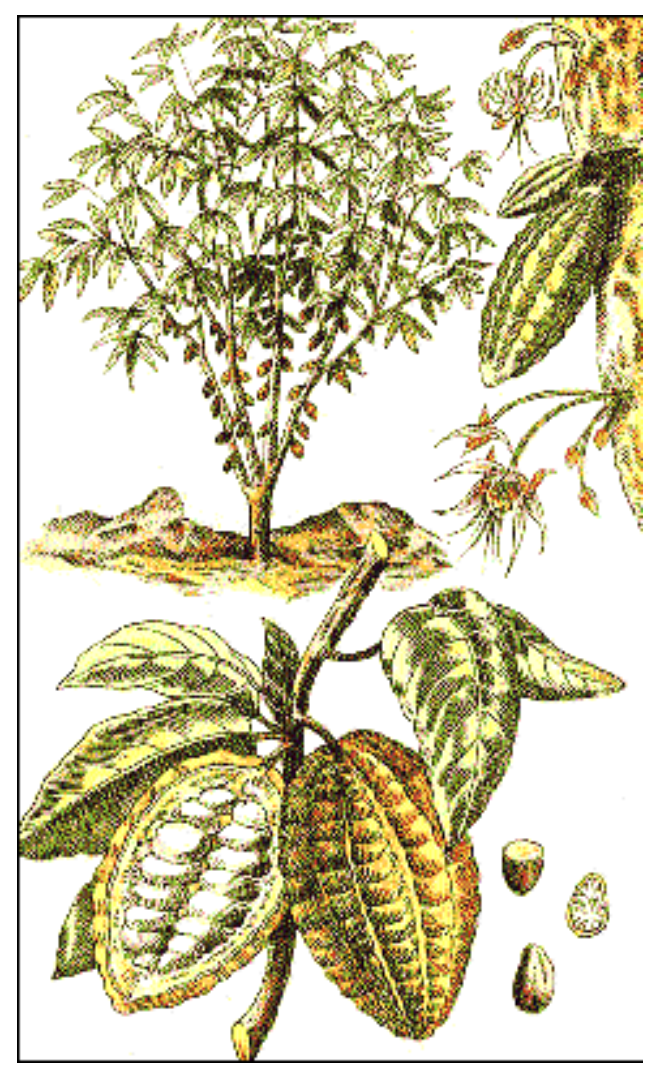

Photo. Cabosse. 


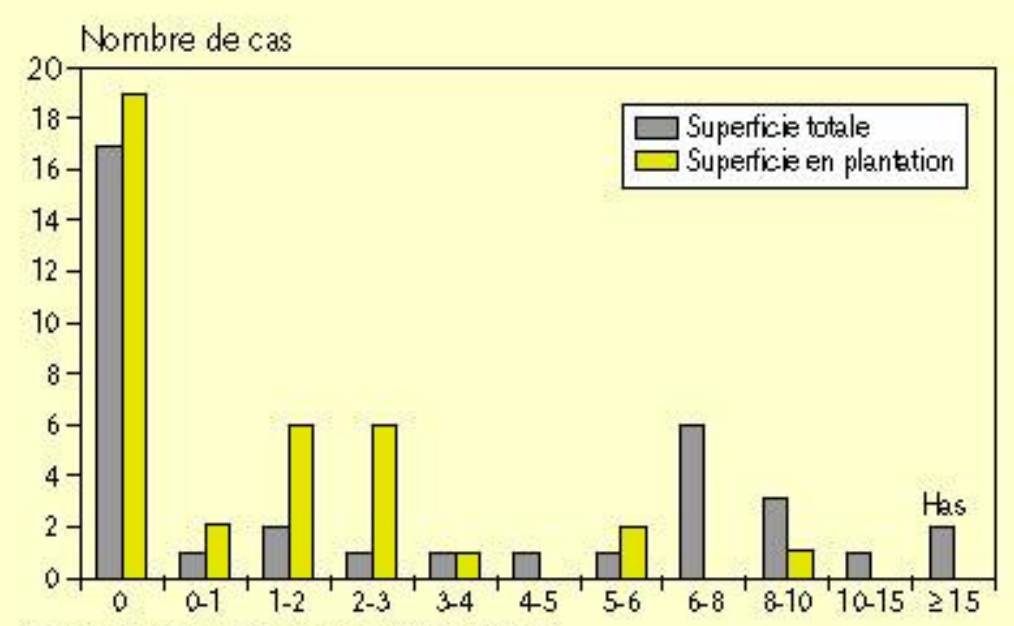

Source: enquutes de l'auteur, avril 1984-juin 1985.

Figure 1. Situation foncière des planteurs de cannabis sur les anciens fronts pionniers de l'Ouest du Sassandra.

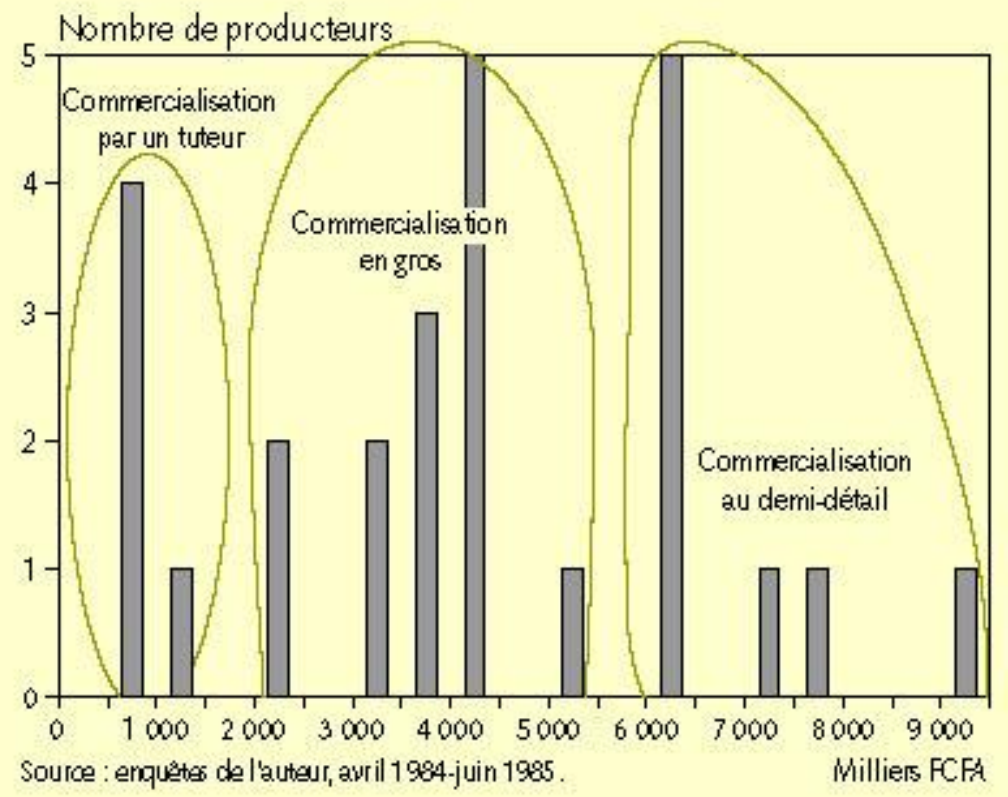

Figure 2. Répartition des revenus obtenus de la culture du cannabis selon les conditions d'accès des producteurs au marché. 